\title{
Dinámica interna familiar como espacio educativo para la construcción de ciudadania*
}

José Wilmar Pino Montoya

https://orcid.org/0000-0001-9035-2058 Universidad Católica Luis Amigó,

Colombia

Jose.pinomo@amigo.edu.co

Juan Fernando López Correa

https://orcid.org/00oo-0002-5258-0997

Universidad Católica Luis Amigó,

Colombia

juan.lopezco@amigo.edu.co

\section{Adriana María Gallego Henao}

https://orcid.org/oooo-0003-2952-1603 Universidad Católica Luis Amigó, Colombia

adriana.gallegohe@amigo.edu.co

\section{Resumen}

Este artículo se propone evidenciar la relación existente entre la dinámica interna familiar y algunos elementos que configuran un ambiente adecuado para educar a los sujetos en aptitudes ciudadanas: la autoridad, los límites y las normas y los roles. El método utilizado es el paradigma interpretativo o histórico-hermenéutico, con un enfoque cualitativo; la estrategia es documental y la ficha bibliográfica y de contenido, como instrumentos privilegiados para la recolección de la información. Para el análisis se utilizó una matriz categorial. Se concluye que los elementos estudiados de la dinámica familiar constituyen un ambiente determinante para la formación de competencias ciudadanas que definen comportamientos cívicos.

\section{Palabras clave (Fuente: Tesauro de la Unesco)}

Ambiente educacional; educación cívica; educación del carácter; educación familiar; medio de aprendizaje; relación padres-escuela.

* $\quad$ El presente texto es derivado del macroproyecto de investigación "Dinámica interna familiar con relación a la participación ciudadana" financiado por la Vicerrectoría de Investigaciones de la Universidad Católica Luis Amigó.

Recepción: 31/07/2019 | Envío a pares: 23/10/2019 | Aceptación por pares: 04/11/2019 | Aprobación: 06/11/2019 


\title{
Internal Family Dynamics as an Educational Opportunity for Constructing Citizenship*
}

\begin{abstract}
This article aims at demonstrating the existing relationship between internal family dynamics and some elements that make up an appropriate environment to educate individuals in citizen skills: authority, limits, rules, and roles. The method used is the interpretive or historical-hermeneutical paradigm, with a qualitative approach. Strategy is documentary and bibliographic and content sheets are employed as privileged instruments to collect data. For the analysis, a category matrix was used. It is concluded that the studied elements of family dynamics constitute a determining environment to develop citizen skills that define civic behaviors.
\end{abstract}

\section{Keywords (Source: Unesco Thesaurus)}

Educational environment; civic education; character education; family education; learning environment; parent school relationship. 


\section{Dinâmica interna familiar como espaço educativo para construir cidadania*}

Resumo

Este artigo propõe a evidenciar a relação existente entre a dinâmica interna familiar e alguns elementos que configuram um ambiente adequado para educar os sujeitos em aptidões cidadãs: autoridade, limites, normas e papéis. O método utilizado é o paradigma interpretativo ou histórico-hermenêutico, com uma abordagem qualitativa; a estratégia é documental e emprega-se a ficha bibliográfica e de conteúdo como instrumentos privilegiados para coletar a informação. Para a análise, foi utilizada uma matriz categorial. Conclui-se que os elementos estudados da dinâmica familiar constituem um ambiente determinante para formar competências cidadãs que definem comportamentos cívicos.

\section{Palavras-chave (Fonte: Tesauro da Unesco)}

Ambiente educacional; educação cívica; educação de caráter; educação familiar; meio de aprendizagem; relacionamento pais-escola.

\footnotetext{
* Este texto é derivado do macroprojeto de pesquisa "Dinámica interna familiar con relación a la participación ciudadana" ("Dinâmica interna familiar com relação à participação cidadã”), financiado pela Vice-reitoria de Pesquisas da Universidad Católica Luis Amigó.
} 


\section{Introducción}

Desde el inicio de las civilizaciones las sociedades han procurado construir pilares que favorezcan la sana convivencia y la armonía de su comunidad, garantizando de esta forma permanecer y crecer en el marco de unas normas que rigen la tranquilidad del clan. De esta manera "la sociedad aparece como un ser colectivo, de naturaleza diferente a la del ser individual, y por lo mismo que preexiste y sobrevive [...] les impone normas y coerciones ineludibles en sustancia" (Azevedo, 2013, p. 69). Bajo esta premisa, se han desarrollado diferentes sistemas políticos que han planteado distintas formas de entender la relación entre los sujetos y la de estos con el Estado. Sin embargo, la preocupación se ha fijado en el desarrollo de estrategias que posibiliten la vida y el progreso, no solo de los individuos, sino también de los pueblos y de la comunidad en general. De allí que la relación entre Estado y ciudadano sea un tema de especial interés para las ciencias sociales, en la medida en que se constituye como un campo de reflexión y producción académica, máxime cuando, según Avendaño y Paz (2016), la sociedad actual está en una verdadera crisis en la que los problemas sociales y éticos son cada vez mayores y buscan ser resueltos desde los procesos de formación.

Acorde con lo anterior, es importante resaltar que es en el núcleo familiar donde se gestan las bases para el desarrollo y la formación como seres humanos. Es con los integrantes de la familia con quienes se aprende la importancia del reconocimiento de las capacidades y las potencialidades de sí y de los otros, ya que con los adultos y personas significativas se desarrollan las competencias y actitudes para actuar e interactuar en la sociedad. De este modo, "cuando la familia le brinda al niño seguridad y permite que exprese todos sus sentimientos, pensamientos y emociones, está contribuyendo a que ese ser que se encuentra en desarrollo logre formarse como ser autónomo, libre e integral" (Gallego, Pino, Álvarez, Vargas y Correa, 2019, p. 4). Es así que la familia, como primer agente de socialización, en palabras de Berger y Lukmman (1978), es la llamada a brindar las primeras bases educativas e interactivas del niño para ser, hacer y convivir consigo mismo, con el otro y con los otros; es decir, es en la familia donde el niño empieza a tomar conciencia de sí mismo y de todo lo que lo rodea.

Estas interacciones le permiten considerarse único, singular y diverso y fortalecen su autoestima, la cual, con el tiempo, lo llevará a madurar, formarse y plantearse un proyecto de vida, desarrollar la capacidad de afrontar los retos que le impone el contexto y constituir su ser ciudadano. Por ello se podría estar de acuerdo con lo afirmado por Gallego, al decir "que la familia es clave en el proceso de socialización primaria debido que las actitudes, la estabilidad, la seguridad, los modos de ser y el cultivo de las diferentes dimensiones se forman en los primeros años vida" (2012, p. 331).

Teniendo como base los anteriores argumentos, se presenta la necesidad de saber qué aspectos de la familia determinan o predisponen a sus integrantes para que, cuando estos sean adultos, participen activamente del espacio político, y cómo estos construyen las competencias para entrar en la vida pública. Es decir, cómo ejercen su ciudadanía, entendida esta, tal y como la presenta Urquijo, para quien "un ciudadano -o una ciudadana- es un agente o 'promotor dinámico de transformaciones sociales' conducentes a superar las situaciones de desigualdad social, exclusión y pobreza. Esto implica participación en los ámbitos político, económico, y civil" (2007, citado por Rincón, 2012, p. 119).

Por esta razón la revisión temática, aquí planteada, surge en el contexto del proyecto de investigación "Relación entre la dinámica interna familiar y la participación ciudadana". Este proyecto se propone y cobra interés por el seguimiento a jóvenes de octavo grado de la Institución Educativa de Villa del Socorro de la Cuidad de Medellín, en quienes se les detectó una dificultad para tomar decisiones autó- 
nomas, participar en estamentos de orden institucional y cumplir así su papel como ciudadanos. Por otra parte, la investigación surge considerando los reportes estadísticos del observador de convivencia de la Institución, donde se encontró que el $65 \%$ de los estudiantes han presentado comportamientos relacionados con intolerancia, agresiones físicas, verbales y apatía por la norma, así como también desánimo para participar en las actividades de aula o de la institución.

Es por ello que los aspectos enunciados en líneas anteriores motivaron esta revisión temática de la dinámica interna familiar y la relación de esta con la construcción de ciudadanía, en la medida en que se piensa que es en la familia donde se adquieren las primeras bases de la socialización y, de hecho, ella es la primera agencia donde el niño y la niña aprende a interactuar, afirmarse y ser parte de lo social. Así nació la pregunta: ¿si la familia es la primera agencia de socialización en la que los niños y las niñas interactúan con libertad, por qué se presentan comportamientos agresivos, autoritarios, violentos y poco participativos en su interacción en el ámbito social? En este orden de ideas, en un primer momento se expone el concepto de familia enmarcado en el proceso de socialización, a la luz de diferentes estudios. En segundo lugar, se discute sobre la dinámica interna familiar haciendo hincapié en algunos de sus componentes, a saber: la autoridad, los roles, los límites y las normas. Y, en tercer lugar, se profundiza en el concepto de ciudadanía y las implicaciones teóricas que conlleva su apropiación y posterior aplicación en los procesos formativos que permitan su construcción. Por último, se ofrecen las conclusiones a las que se llega con el rastreo documental.

\section{Método}

Para el desarrollo de esta investigación se asumió el paradigma cualitativo de enfoque hermenéutico, que se entiende como un proceso interpretativo de indagación que examina un problema humano o social. Se acudió a la estrategia de investi- gación documental "conformada por proposiciones teóricas específicas a la parte de la realidad social que se pretende estudiar" (Sautu, Boniolo, Dalle y Elbert, 2005, pp. 34-35). De allí que esta investigación parte del análisis hermenéutico y se fundamenta en la interpretación de textos y material literario, es decir, tiene el propósito de descubrir el significado de las palabras, los escritos y los textos consultados (Galeano y Vélez, 2000). Las categorías que guían el rastreo teórico y epistemológico son: familia, dinámica familiar y construcción de ciudadanía. De los 120 textos encontrados, solo se abordaron los 50 que respondían a los criterios de inclusión, tales como: autores clásicos y modernos, así como investigaciones que no excedieran de los 7 años y que respondieran a las categorías del estudio. Por otra parte, se incluyeron artículos de investigación teóricos, estados del arte y empíricos, de enfoque cualitativo y cuantitativo o mixtos, que claramente se centraran en la relación entre la familia y la socialización, entendiendo la familia como agente socializador y como espacio para la construcción ciudadana. Fueron excluidos documentos de páginas web sin sustento retórico claro o sin evidencia de haber pasado por un proceso de evaluación; además no se tuvieron en cuenta aquellas revistas que no ostentaran un proceso arbitrado para la publicación de sus artículos. Las bases de datos consultadas se precisan en artículos que se publicaron en Google Académico en bases de datos como: Scielo y Ebsco, Latindex y Redaly c. Por otra parte, se consultaron publicaciones de bibliotecas y centros de documentación de las universidades públicas y privadas de la ciudad de Medellín. Paso seguido, se realizó la sistematización y depuración de la información en fichas de contenido, las cuales indagaban por los aportes más significativos y pertinentes para el desarrollo de la presente investigación. Finalmente, se procedió a la compilación del texto atendiendo a la posibilidad de acercarse comprensivamente al papel de la familia y a la forma como se desarrolla su dinámica interna con respecto a la construcción de ciudadanía. 


\section{Resultados y discusiones}

\section{Concepciones históricas de familia}

Históricamente el concepto de familia ha ofrecido un amplio abanico de formas de comprensión que fundamentan su sentido sobre la base de diversas perspectivas, dinámicas, cambios en su estructura, composición y múltiples transformaciones de la sociedad moderna. Según Gallego (2012), si bien estas características constituyen un problema a la hora de plantear una definición unificada de la familia, a su vez contribuye a describir las características y finalidades de este grupo social conforme a la realidad de las distintas problemáticas que se forman en la actualidad. Es de tener en cuenta, además, que la concepción de familia ha sido abordada por múltiples disciplinas, como la antropología, la economía, la demografía, la ciencia política, la antropología cultural, la sociología y la psicología. Para fines de este estudio, se asumen las reflexiones, concepciones y planteamientos de la teoría sociológica, por cuanto esta perspectiva centra su objetivo en grupos más heterogéneos y complejos, propios de las sociedades modernas.

Para la sociología, en palabras de Gallego (2012), la familia puede entenderse como la base sobre la cual se construye la sociedad. Esta aseveracion la constata la Constitución Política de Colombia al definir la familia como "el núcleo fundamental de la sociedad que se constituye por vínculos naturales o jurídicos" (art. 42). Como se aprecia en la anterior definición, la familia tiene su origen en la misma naturaleza, lo que le suministra una vinculación biológica a la relación de sus integrantes; por otra parte, también se la entiende como acuerdo voluntario de las partes, que deciden formalizar a través de un documento jurídico, una ley o una norma. En este sentido, el concepto de familia denota el carácter vinculante de su ser y su esencia en la vida de un Estado y la sociedad en general. Según Bezanilla y Miranda, "a la familia se le considera el grupo primario por excelencia, debido a que la persona, desde su naci- miento, se encuentra inmersa en él y es ahí donde vive y desarrolla las experiencias y habilidades que servirán como base para la vida en todos los ámbitos de su existencia" (2014, p. 60).

Para la jurisprudencia colombiana, entonces, la familia constituye el primer centro de socialización del individuo, en el que se empiezan a constituir, a partir de las relaciones iniciales con los suyos, la asimilación de las normas y el respeto por el otro, en aras de una convivencia sana mediante la cual todos sus miembros puedan desarrollar las capacidades fundamentales para la adquisición de las competencias ciudadanas. Así que la sociología, según Estrada (2007), ha definido la familia como un grupo social en el que convergen los individuos, que tienen una residencia común y una organización propia, donde interactúan de acuerdo con sus respectivas posiciones, estatus, responsabilidades y roles específicos, buscando mediante su interacción unos fines más o menos designados. También desde el punto de vista sociológico se entiende la familia como "un grupo de personas entrelazadas en un sistema social, cuyos vínculos se basan en relaciones de parentesco fundados en lazos biológicos y sociales con funciones específicas para cada uno de sus miembros" (Páez, 1984, p. 23). Esta perspectiva secunda la afirmación de la familia como punto de partida de la sociedad, a partir de la cual se establecen unas relaciones que empiezan por ser de sangre o afinidad, pero que luego se amplían a unos vínculos más complejos en los que la diversidad y la diferencia son la característica fundamental. De allí que a la familia se la defina como una realidad vinculante y potente en el desarrollo humano, social y político de los individuos o como estructura social fundamental en la generación y potenciación de competencias para participar en procesos sociales. Esto la hace garante de cuidado del Estado, de las diferentes entidades públicas y privadas, entre las que se encuentran las instituciones educativas, que deben prestar una mayor atención a su configuración, conservación y relación, 
debido a que es en ella donde se inicia el entramado social en que los seres humanos empiezan a entretejer sus relaciones sociales y su capacidad de actuar y cuidar de los otros.

Consciente de esta realidad, el Municipio de Medellín define la familia como "un sistema social, viviente, dinámico y complejo en donde emergen relaciones que la mantienen unida y posicionan como una red social primaria. Implica la existencia de vínculos de parentesco (afinidad y/o consanguinidad) entre sus integrantes" (2010, p. 5). Desde esta perspectiva, la familia se convierte en la base para que los individuos adquieran desde que nacen la capacidad de asumir su papel en la sociedad, aspecto en el que coinciden Infante y Martínez (2016); Vergara (2017); Alzate (2003); Froufe (1995) y Álvarez (2010), al afirmar que la familia es el ambiente de crianza decisivo para el desarrollo infantil, pues en ella se cultivan, desarrollan y potencian las habilidades, competencias y comportamientos necesarios para posteriores desarrollos sociales y humanos.

La familia es, pues, un "grupo de personas que comparten vínculos de consanguinidad, parentesco, afecto y convivencia" (Louro, 2005, p. 334), idea que es secundada por Estrada, al afirmar que "las familias pueden tener lazos de consanguinidad, afinidad y adopción y [que] a partir de estos vínculos se generan distintas formas de organización familiar" (2007, p. 6o). Pero también puede ella ser conformada por vínculos jurídicos o por alianzas o pactos que se tejen entre las personas integrantes (Sánchez, Aguirre, Solano, y Viveros, 2015). Dicho lo anterior, podría plantearse que en la idea de familia no solo subyace la definición planteada de unión de un hombre y una mujer que contraen matrimonio con la voluntad de crear una familia (Constitución Política de Colombia, art. 42), sino que también trasciende a otras esferas más generales y vinculantes, en las que se amplía el concepto, más allá de los lazos de sangre.

En suma, la familia, como agencia de socialización primaria, tiene la tarea de formar y preparar a sus integrantes para ser sujetos políticos, ciudadanos integros y seres humanos felices. Moreno, Estévez, Murgui y Musitu "sostienen que el clima familiar es uno de los factores de mayor relevancia en el ajuste psicosocial de los hijos; ejerce una influencia significativa tanto en la conducta, como el desarrollo social, físico, afectivo e intelectual de los integrantes" (2009, citados por Sánchez y Valdez, 2011, p. 183). De ahí la importancia de pensar la familia como base social, debido a que es la estructura fundante de individuos independientes, además que permite el desarrollo mediante la identificación de sí mismo y del otro en un entorno llamado sociedad.

\section{La familia y su función como agente socializador}

La familia se convierte, entonces, en un espacio de socialización decisivo en la formación democrática y ciudadana y se entiende como una forma de convivencia que prepara a sus miembros para relacionarse con otros, vivir en sociedad y ejercer la ciudadanía. Esta concepción reconoce entonces su función productora de cambio social (Zuluaga, 2004), así como las funciones biológicas, económicas y afectivas (Vásquez, 2005), ámbitos en los que responde a una serie de necesidades, que empiezan por las de crecimiento y desarrollo de los niños y las niñas y por sentirse amado y protegido, y se le suman "autonomía personal, adecuada socialización, responsabilidad y sentido de su vida" (Reusche, 2011, p. 9). En este sentido, se podría decir que los numerosos intercambios que se dan entre la familia y el entorno la determinan como un sistema abierto que asegura la reproducción de las generaciones, pero también los procesos de socialización de los niños y las niñas (Zuluaga, 2004). Así, entonces, entenderíamos la familia como "el grupo responsable de cuidar y proteger al nuevo ser que nace, pero también de integrarlo al mundo y de hacer que, a través de la enseñanza de prácticas, reglas y pautas de convivencia, pueda adaptarse exitosamente a las necesidades de la sociedad" (Yabal, 2017, p. 10). 
En el enfoque sistémico, "la familia no puede concebirse como un elemento en el que todas sus partes actúan individualmente o toman sus decisiones de manera aislada de las otras partes que la configuran" (Pino, 2012, p. 161). Desde esta perspectiva, la familia debe entenderse como un todo, en aras de su atención, y no solamente partiendo de uno de sus miembros, debido a que entre ellos existen cantidades de relaciones y vínculos que determinan la aparición de las conductas humanas. Al respecto, Gimeno señala que "la familia es un todo interrelacionado, en el que la decisión de una de sus partes trasciende a todas las demás, en tanto en la familia conforman un todo integrado" (1999, citado por Pino, 2012, p. 112).

Siguiendo esta línea, Estrada la define como "una célula social, cuya membrana protege en el interior a sus individuos y los relaciona al exterior con otros organismos semejantes" (2007, p. 60). El autor en mención ratifica que mediante la interacción de sus miembros se posibilita el proceso de socialización, en el que estos se reconocen como parte de un grupo particular que se halla inmerso en otro más general. Así, de tal modo, "la familia debe asegurar la creación de vínculos afectivos que funjan como precursores de otros; de manera que la unidad familiar otorga fuerza y sentido a sus miembros desde el interior, pero a la vez los relaciona y pone en contacto con el exterior" (Gil, 2007, p. 18).

En este sentido, el espacio familiar está demarcado por aspectos como la asimilación valorativa de los derechos, deberes, responsabilidades y obligaciones que guían y forman los patrones de comportamiento que la sociedad necesita en la construcción de los futuros ciudadanos. Como se puede deducir de lo anterior, "la familia es un espacio de aprendizaje de valores y normas de conducta social y de orientación política" (Rincón, 2012, p. 121). Así, ella debe ser contextualizada en virtud de las relaciones e interacciones sociales que posibilitan a sus miembros el desarrollo de competencias emocionales y ciudadanas que respondan a las necesidades de la sociedad.
Esto es factible en cuanto que en el grupo familiar se dan ciertas dinámicas que permiten desarrollar las capacidades sociales de los sujetos.

\section{Dinámica familiar y el proceso de socialización}

El concepto de dinámica familiar tiene diferentes acepciones que constituyen las variadas formas estructurales y comportamentales de la familia. Al respecto, Torres et al. precisan:

La dinámica familiar son las fuerzas intrínsecas que se movilizan en un sistema familiar produciendo relaciones y comportamientos particulares. Asi pues, la manera en la que una familia vive e interactúa unos con otros es lo que crea la dinámica. Existen muchos factores diferentes que aportan y/o influyen en una dinámica familiar, como el número de integrantes de ese sistema, la definición o no de la jefatura de hogar, aspectos de ciclo vital del grupo familiar; valores y creencias; prácticas en relación con el cuidado de los hijos; asi como otros factores económicos $y$ sociales. (2014, p. 9)

Para Rondón (2011), en estas dinámicas y según las múltiples características de las familias, no solo se puede decir que dentro de este grupo se comparten unas necesidades afectivas, sino que también se dan unas funciones compartidas y negociadas por sus miembros. Esta idea permite entender a la familia como célula básica del cuerpo social, en la que, a través de las formas de relación, se construye ciudadanía, sin importar el tipo o la clasificación a la que pertenezca. La dinámica familiar posee una serie de características que determinan la comprensión de la misma, lo cual

... obliga a conocer la Jerarquía entendiéndola como el nivel de autoridad que gobierna en la organización familiar y que puede ser autoritaria, indiferente, negligente, indulgente, permisiva o reciproca con autoridad. Los límites que representan las reglas que delimitan a las jerar- 
quías y que pueden ser claros, difusos o rígidos, y la comunicación, que no es más que la forma en que la familia se expresa entre sí, y puede ser, directa, enmascarada o desplazada. (Mendoza et al., 2006, p. 28)

La dinámica interna familiar involucra diferentes procesos que están determinados por factores biológicos, sicológicos y sociales y responden a la condición natural que busca conservar la especie (Alcaldía de Medellín, 2010). De tal modo, la dinámica familiar tiene unos componentes que van a ser determinantes a la hora de orientar los comportamientos y las relaciones entre los miembros. Según la Alcaldía de Medellín, entre ellos están: "la afectividad, la comunicación, la autoridad, las normas, los roles, los límites y el uso del tiempo libre. Lo anterior, enmarcado en el cumplimiento de la función socializadora" (p. 13). El anterior argumento lo complementan Infante y Martínez (2016), quienes arguyen que a partir de la dinámica familiar y las experiencias que viven los individuos dentro de este núcleo se va desarrollando el proceso de socialización y los primeros aprendizajes del individuo, por ejemplo a reconocerse dentro del grupo y a desempeñar un rol en el que aprenderá un sistema de valores determinados por la crianza.

Teniendo en cuenta la importancia que cobra la dinámica familiar en los procesos de construcción ciudadana, se analizarán tres de los componentes, a saber: la autoridad, el rol de los miembros y los límites, como elementos que permitirán establecer una relación entre la familia y la construcción de ciudadanía.

\section{La autoridad: una necesidad natural del orden social}

La familia es el primer espacio de interacción social en el que el individuo se relaciona con la autoridad, determinada por un ámbito generacional y de respeto en el que se circunscriben los hijos a la voluntad de los padres, en la que estos ejercen el poder. Como bien lo sustenta el siguiente aparte:
La jerarquía refleja el modo en que el poder y la autoridad se distribuyen dentro de la familia. Un sistema funcional se organiza jerárquicamente; el manejo y distribución del poder en la familia explica su organización jerárquica. El poder es la capacidad de influencia que tiene un individuo determinado para controlar la conducta de otro; idealmente el poder debe estar en manos de la persona que ocupa una posición de autoridad. (Robles, 2017, p. 17)

La Alcandía de Medellín (2010) afirma que este poder les permite a los padres o sus sustitutos cumplir ciertas funciones encaminadas a la dirección, protección, educación y formación de los hijos, cuyo objetivo es propiciar un ambiente sano en el que la familia se pueda desarrollar y los hijos, de manera especial, asimilen e incorporen a su comportamiento habitual las normas y los valores de la sociedad.

La autoridad es el eje conductor que integra y guía el cumplimiento de todas las funciones familiares que debe llevarse bajo el control de ambos padres, si los hay, o por un sustituto que dinamice las relaciones en el subsistema parental-filial. Según Góngora, "autoridad significa sostener para crecer. Está caracterizada por el respeto mutuo (padres hijos); se fundamenta en la consistencia, la firmeza, la paciencia y el cariño. Es determinante para la vida de familia y significa la puesta en marcha de los derechos y obligaciones, tanto de hijos como de padres, de una manera equilibrada y flexible" (2006, citado en Jurado, 2013, p. 55).

Los padres, entonces, son quienes marcan los ritmos del entorno familiar a partir de una relación recíproca de respeto en la cual los hijos aprenden a relacionarse con la norma y con quienes tienen la posibilidad de hacerla valer.

Mediante un estilo democrático (de la autoridad), los padres están promoviendo la cooperación de sus hijos, la responsabilidad, la iniciativa, el entendimiento de la pertinencia de las nor- 
mas, la comprensión de la necesidad del respeto hacia el otro y una mayor madurez social y moral; tal estilo fomenta una verdadera interiorización de las normas. (Sánchez y Valdez, 2011, p.182)

Este elemento es fundamental para desarrollar la construcción de ciudadanía, que se da en la medida en que se aprenda a convivir con el otro, en el reconocimiento de la diversidad y la diferencia de cada individuo y en correspondencia con la norma, como límite de la libertad, la cual permite el desarrollo de todos en igualdad de oportunidades.

La vivencia del derecho y el deber es el rol que desempeña el ciudadano que se asume como miembro de un colectivo que busca el desarrollo de todos y construye, fundado en su comprensión, una mejor sociedad. En esta línea, los derechos y deberes implican:

... la vida en democracia, incluyendo la participación responsable en las actividades comunitarias y ciudadanas, que reconozcan la legitimidad de distintos puntos de vista, los que reflejan la diversidad de visiones que conviven en el pais, que actúen responsablemente consigo mismos, con su entorno y en la sociedad, y que valoren los principios fundamentales de libertad, igualdad, justicia, pluralismo, responsabilidad social y respeto a los derechos humanos. Asimismo, busca que valoren la dignidad de todos los seres humanos entendiendo a la persona como sujeto libre, autónomo, dotado de derechos y deberes. (Decretos 256 de 2009 y 254 de 2009 , citados por Bornand, 2011, p. 39)

En esta medida, es fundamental entender la manera como los individuos asumen la norma, pues ello permitirá concebir si esta incide o no en la construcción de ciudadanía. "La disciplina, al imponer hábitos, regula y contiene las conductas humanas transformándolas en conductas normalizadas, lo que según Durkheim no implica la pérdida de la libertad, en tanto la define como fruto de la reglamentación que posibilita ordenar la conducta hacia el logro de los ideales humanos" (Alvarado y Botero, 2009, p. 6).
En otras palabras, la autoridad es fundamental en los procesos de formación que se den dentro del hogar y posibilita el proceso de adaptación de los hijos a la vida cívica, en la medida en que reconocen la autoridad del Estado y de la ley como elementos fundantes de la vida en sociedad. López, Murgui, Moreno y Musitu aclaran un poco más la anterior apreciación al afirmar que "en las familias donde se ejerce una autoridad asertiva y democrática los niños presentan actitudes favorables hacia la autoridad institucional", prueba de "un auto concepto general más positivo, en comparación con aquellos que muestran actitudes negativas hacia la autoridad formal" (2007, citados en Sánchez et al., 2015, p. 130).

\section{Los límites y las normas: punto de partida para el ejercicio de la libertad}

Los límites son aquellas regiones o sectores que sirven de barrera de diferenciación entre los miembros de una familia. Son el espacio en el que se permite la protección, sin perder la individualización y diferenciación de quienes conforman el grupo familiar (Alcaldía de Medellín, 2010, p. 15). A partir de la experiencia de los límites, se logra la vivencia del respeto y el reconocimiento del otro, en la medida en que confronta aquello que se me permite con lo que no está permitido. Los límites posibilitan el uso de la libertad, enmarcada en un espacio donde caben otros, con las mismas posibilidades, aunque no iguales ni con los mismos intereses. Esta relación que se establece dentro de la familia permite que el sujeto empiece a entender que su propio desarrollo está ligado al desarrollo de los demás y que las libertades individuales están sujetas a las libertades de otros sujetos que como él buscan su realización. En este sentido, el límite y la norma permiten la comprensión del otro y de lo otro, comprensión que construye ciudadanía, por cuanto lleva al entendimiento de la diferencia. De acuerdo con Richardson, los comportamientos de los niños y las niñas están permeados por los límites y marcados por los padres y las expectativas de comportamiento de los infantes en los diferentes ambientes (1993, citado en Gallego, 2012, p. 335). 
Por otro lado, los límites establecen la disciplina y el orden como garantías del buen funcionamiento de la vida familiar y sientan las bases de positivos comportamientos futuros, tanto individuales como sociales. En este caso, según Gallego, los comportamientos de los miembros de la familia "están permeados por los límites, marcados por los padres y las expectativas de comportamiento de los infantes en los diferentes ambientes, así como por las reglas de convivencia, las costumbres, los valores y los intercambios afectivos constantes" (2011, citada por Sánchez et al., 2015, p. 130).

Para Cuervo (2010), el establecimiento de la norma se da a través de los límites que se establecen en el hogar, que se definen como la pauta que determina el comportamiento de un sujeto ante las diferentes circunstancias que se le presentan y que le exigen una toma de posición. La importancia de las normas y de los límites es que son educativas, forman al ser humano para la vida en sociedad, debido a que este no nace sabiendo cómo debe comportarse; al contrario, nace siendo inmaduro, impulsivo, insaciable y sin disciplina.

En coherencia con lo expresado en líneas anteriores, los padres o adultos significativos deben estar convencidos de su función educativa, en la cual es necesario establecer límites y normas que redunden en la sana convivencia, tanto en la familia como en el escenario educativo. Y es que "las crecientes demandas de expresión y afirmación de una singularidad, que ya no reconoce los límites impuestos por códigos acatados colectivamente, llevan al debilitamiento del rol social, lo que tiene consecuencias sobre las formas de socialidad" (García, Martínez, Moreno y Armenta, 2010, p. 124).

Por otra parte, los límites y las normas son fundamentales, porque generan en los hijos sentimientos de seguridad y protección, a la par que van creando sus propios referentes y adquiriendo conocimiento de lo que es y lo que no es válido, lo cual les ayudará a ir estructurando su propia escala de valores. A propósito, y según Torres et al., "los límites incluyen un set visible de lealtades, reglas y de conexiones emocionales". Estos límites pueden ser "internos, entre los sujetos que conforman el grupo familiar", o externos, "entre la familia y los grupos e instituciones sociales con que interactúan" (2015, p. 227). Además, fortalecen la capacidad de los hijos para lograr una convivencia más organizada y promueven el respeto hacia los demás y hacia sí mismos. Como afirman López, Galván y Blanquiceth, los límites "son importantes en la medida [en] que generan responsabilidad, conciencia crítica, madurez y compromiso frente a muchas situaciones" (1999, citados por Sánchez et al., 2015, p. 132). En conclusión, y como lo dice Cuervo (2010), los límites y las normas preparan a los hijos para la vida en sociedad que se rige por restricciones y obligaciones que deben aprender a cumplir.

\section{Los roles: el despertar de la autonomía y la responsabilidad}

Ahora es preciso aclarar el concepto de rol. Según López, acá está en juego "la adquisición del rol y la identidad sexual: función de la familia". Por ello señala que "la división de roles se da a partir del género", entendiendo por rol sexual "los comportamientos, sentimientos y actitudes que se consideran propios del hombre o de la mujer". De este modo, el concepto de rol en la familia tiene una base cultural (1984, citado por Gallego, 2012, p. 337). Por su parte, Viveros y Arias definen rol como "el comportamiento esperado de una persona que adquiere un estatus particular. Cada persona puede ocupar varios estatus y puede esperarse que desempeñe los roles apropiados a ellos" (2006, citados por la Alcaldía de Medellín, 2010, p. 15).

Por este motivo y a pesar de que la familia puede entenderse como un todo, como un equipo, eso no quiere decir que todos sus miembros sean iguales; precisamente en la diversidad radica la gran riqueza humana de este núcleo, que posibilita que cada miembro se desenvuelva dentro del hogar 
cumpliendo un rol diferenciado. "La función de roles mantiene la estabilidad del sistema familiar. El suprasistema engloba todos los sistemas de la familia y todos con los que se tiene contacto como trabajo, colegio y amigos" (Sánchez et al., 2015, p. 22).

De lo anterior se deduce que es necesario que cada miembro de la familia desempeñe un rol o unas funciones específicas que, al ser realizadas, se convierten en modelo, en la medida en que generan comportamientos por imitación. Así, los hijos aprenden de los padres y los hermanos menores de los mayores (Cuervo, 2010), y de esta manera se apoyan y ayudan, con lo que generan que las prácticas familiares cumplan una función pedagógica y formativa, labor que termina fortaleciendo el ideal de vida de los hijos y permite que vayan descubriendo en sí mismos aquello en lo que quieren realizarse y esbocen un primer proyecto de vida mediante el cual entenderse en relación ellos mismos y con el entorno. Vásquez (2005) propone que los roles deben ser asumidos de manera auténtica y asertiva, aceptando las consecuencias positivas y negativas inherentes a ellos; en esta medida, la familia se convierte en un elemento mediador entre el niño y el entorno en el que vive, y juega un papel sumamente importante que incidirá en el desarrollo personal y social.

Parte importante del rol de los padres, dice Cuervo (2010), es la asignación equitativa de responsabilidades entre los hijos, según las etapas de crecimiento y desarrollo. Por ello hay que estimular tempranamente en el niño todas las habilidades y potencialidades posibles, para que, al asumir su vida, sienta la satisfacción que depara actuar con autonomía.

El rol es un elemento que demarca la posición o estatus entre los miembros del sistema familiar. Es el vínculo que el individuo posee para comunicarse y enfrentarse con el mundo cumpliendo con diversas funciones, deberes y derechos que han sido introyectados en el núcleo familiar durante el desarrollo bio-psico-social de cada miembro. El rol equivale a las expectativas de conducta que son dirigidas hacia una persona en una situación o en un determinado contexto. (Frachia, 2015, p. 19)

Así, los hijos también asumirán distintos roles individuales específicos, de los cuales deben apropiarse asumiendo las responsabilidades familiares que deben cumplir. Estos elementos fundamentarán el proceso de socialización y los preparará para una vida cívica y ciudadana en la que todos puedan desarrollar sus potencialidades a partir de su propio reconocimiento y el de los otros.

\section{El lugar de la familia en la formación ciudadana}

El hombre, entendido como el zoo politikón de Aristóteles, apunta a determinar elementos que posibiliten de mejor forma su existencia, y es a esta construcción a lo que se llama ciudadanía, resultado de años de evolución social y política. En esta mirada, hablar de ciudadanía implica ahondar en los procesos de formación de los sujetos dentro de la estructura del Estado. Implica pensar en valores éticos, en formar personas respetuosas de lo público, que se apropien de la importancia de la vivencia de los Derechos Humanos, que asuman su rol ciudadano cumpliendo con sus deberes y luchen por sus derechos, procurando una mejor sociedad para todos, en la que se pueda desarrollar la persona y vivir en paz. Por estas razones es fundamental pensar la formación cívica como un componente esencial de la educación (Naval y Ugarte, 2008), pues es la formación del niño y del joven la que hará posible desarrollar la idea de ciudadano como sujeto político. Es aquí cuando resulta de vital importancia precisar la incidencia de la familia en el proceso de socialización y es determinante la manera como se desarrolla la responsabilidad, todo ello para la construcción de la sociedad que se desea, una en donde todos los individuos puedan elegir de acuerdo con las múltiples opciones y oportunidades que se ofrezcan, en condiciones de equidad (García y López, 2014). De este 
modo, se reconoce la familia como generadora de cambio social (Zuluaga, 2004) y su dinámica puede constituir excelentes referentes de construcción de ciudadanía, en la medida en que una familia que establece relaciones democráticas y su práctica se fundamenta en el ejercicio de derechos y deberes estará en mejores condiciones para fortalecer y apoyar el desarrollo individual, colectivo y humano de sus miembros (Cebotarev, 1984).

La relación entre familia y ciudadanía se establece a partir de la socialización primaria que tiene lugar, como dice Rincón, "en los ámbitos cotidianos de la niñez, mediada por el lenguaje y las emociones". Se precisa así que "la familia es un espacio de aprendizaje de valores y normas de conducta social y de orientación política" (2012, p. 121) y su situación como espacio de socialización se constituye a raíz de sus funciones referidas de crianza y cuidado de las nuevas generaciones, construcción de identidad individual y reproducción de la identidad social, que Zuluaga define como el resultado "de la configuración de dinámicas de sociabilidad, que le son propias y se diferencian de otros lugares sociales" (2004, p. 90). Por lo anterior, las familias se presentan como el lugar de aprendizaje de niños y niñas pues es allí donde se aprende valores y se desarrollan las competencias ciudadanas que permiten la socialización primaria y posteriormente la secundaria.

En este orden de ideas, se puede pensar que el discurso de la ciudadanía es el de la inclusión, donde todos caben, con sus formas de pensar, ser y parecer; sin embargo, esta inclusión tiene límites, pues así cada uno pretende defender una idea, esta no puede ir en contravía del otro. Con lo anterior, se plantea la importancia de construir ciudadanía, ese "conjunto de conocimientos, habilidades cognitivas, emocionales y comunicativas que, articuladas entre sí, hacen posible que el ciudadano actúe de manera constructiva en la sociedad democrática" (Restrepo, 2006, p. 165), de tal forma que se construya un mundo más justo, en el que cada ciudadano se sienta identifica- do y pueda desarrollarse, desde la diferencia y en el pleno ejercicio de la libertad que le es propia.

La razón de lo anterior radica en la importancia que día a día cobra la formación política y la preocupación social por una construcción de ciudadanía que lleve a convivir con el otro y a construir sociedades más inclusivas y participativas. En consecuencia, la construcción de ciudadanía atraviesa toda la dimensión política, en la medida en que es en la política donde la ciudadanía se concreta y se populariza, obedeciendo a una necesidad por contrarrestar una crisis social (Avendaño y Paz, 2016) que determina la dinámica de las sociedades actuales. Sin embargo, si la construcción de ciudadanía no está garantizada desde la socialización primaria en las familias, la formación cívica se tornará más difícil de lo imaginado. Rojas (2008) afirma que la participación en política es el producto de una construcción social y, por lo tanto, sujeta a cambios, ya que depende de la variación de los significados e imaginarios de las comunidades y, de manera más específica, de las familias.

Por lo anterior, la familia debe constituirse como el lugar privilegiado de construcción de ciudadanía, teniendo en cuenta el papel significativo que juega dentro de las sociedades, pues es evidente que se constituye como lugar de aprendizaje fundamental para la vida en sociedad de los niños y niñas, e incluso es en la socialización primaria donde se implanta con mayor fuerza esas construcciones sociales (Berger y Luckmann, 1978).

En consideración a lo anterior, la familia no es solo el lugar donde se nace; es el lugar propicio donde se desarrolla la vida y el sujeto como persona; es el lugar en el que se posibilita la identidad, se constituye la vida social y se forman las primeras competencias ciudadanas; donde se aprende a resolver conflictos y a negociar, donde se comprende que el mundo no es habitado por un individuo, sino por una colectividad que requiere de límites y normas que permitan que todos los sujetos se puedan desa- 
rrollar conforme a su voluntad, reconociendo al otro en condiciones de igualdad y equidad.

La ciudadanía y la familia no son solo un acto público; son un lugar de construcción de vida y de identidad. Aquí la familia, simbólicamente, es un escenario de vida social, de conflictos, de capacidad de negociación, de reconocimiento de límites, de sentido de lo colectivo, de sentido de responsabilidad pública, para aprender a vivir en comunidad. Ahí radica el sentido y significado de la familia. Pensar en esa experiencia de vida familiar como experiencia socializadora brinda la alternativa de aprender a habitar la vida, a relacionarnos con las demás personas, a vivir la diferencia y el reconocimiento de la diferencia del otro.

\section{Conclusiones}

La construcción de ciudadanía es un tema que cada día cobra más importancia en las democracias modernas y el desarrollo de esta competencia marca en particular las intencionalidades del sistema educativo y en general la formación de los ciudadanos, pues se busca generar transformaciones positivas en la sociedad. Sin embargo, la amplia literatura del concepto posee distintas connotaciones que posibilitan un mayor número de interpretaciones. Se hace necesario tratar de determinar un concepto de validez universal que permita orientar la idea de sociedad enmarcado en los ideales de la democracia, la justicia y la equidad.

Sin embargo, a pesar de la amplia conceptualización acerca de la ciudadanía, hay un elemento común en lo que respecta a su construcción inicial, y es la familia. Esta se reconoce como el elemento fundamental en el proceso de socialización de los individuos y su papel es decisivo a la hora de formar en principios cívicos y democráticos. Es en la convivencia entre sus miembros en donde se preparan los individuos para que aprendan a relacionarse con los otros, a vivir en sociedad y a ejercer la ciudadanía. La familia es, según Zuluaga (2004), un productor de cambio social que favorece o no la construcción de ciudadanía. Así, entonces, la familia se reconoce como el escenario por excelencia donde, a partir de los valores, las normas, las pautas de comportamiento que se orienten, configuran la conducta y proceder del individuo (Sánchez et al., 2015). De acuerdo con las formas como estas interacciones se den en su interior, se determinará el desarrollo de las condiciones que permitan la construcción de ciudadanía, según la forma como interactúe con los otros y la participación que asuma en el entorno social que habita.

Por consiguiente, no solo la familia y su estructura influyen en la construcción de ciudadanía, sino también la dinámica interna familiar, en la medida en que, desde las formas como se desarrollan la autoridad, los límites, las normas y los roles de cada uno de los miembros de la familia, se empieza a desarrollar la ciudadanía. Esto es así porque la dinámica interna familiar desarrolla en sus miembros las competencias básicas para que los ciudadanos sean capaces de reconocer al otro y de asumir la ley, como norma de convivencia que posibilita el desarrollo de la sociedad de forma armónica e incluyente, respetando la diferencia y reconociendo al otro como igual. La dinámica familiar se constituye como referente de construcción de ciudadanía, pues es a partir de las relaciones democráticas y del respeto por los derechos y deberes que cada miembro debe asumir en su hogar como estará en mejores condiciones para apoyar el desarrollo individual y colectivo de sus miembros (Cebotarev, 1984). En otras palabras, la vida cívica y la vivencia de la ciudadanía serán un referente de cómo se asuman la familia y el desarrollo de la vida social. 


\section{Referencias}

Alcaldía de Medellín (2010). Módulo dinámico familiar. Medellín: Programa Medellín Solidaria. Recuperado de https://www.medellin.gov.co/irj/go/km/docs/wpccontent/Sites/Subportal del Ciudadano/Medellín solidaria/Secciones/Publicaciones/Documentos/2011/Cartilla Modulo Familiar.pdf

Alvarado, S. y Botero, P. (2009). Módulo socialización politica y construcción de subjetividad. Documento de Estudio, Maestría en Educación y Desarrollo Humano, Universidad de Manizales - CINDE, Manizales. Recuperado de http://reduei.com/wp-content/uploads/2012/10/Socialización-Política-en-la-formaciónciudadana..pdf

Álvarez, M. (2010). Prácticas educativas parentales: autoridad familiar, incidencia en el comportamiento agresivo infantil. Revista Virtual Universidad Católica del Norte, 31, 253-273. Recuperado de http://revistavirtual. ucn.edu.co/index.php/RevistaUCN/article/view/44

Alzate, M. (2003). El descubrimiento de la infancia. Modelos de crianza y categoría sociopolítica moderna. Revista de Ciencias Humanas, 9(31), 17-24. Recuperado de http://revistas.utp.edu.co/index.php/chumanas/ article/view/889

Avendaño, W. y Paz, L. (2016). Construcción de ciudadanía: un modelo de construcción en la escuela. El Ágora USB, 16(2), 479-492. DOI: https://doi.org/10.21500/16578031.2444

Azevedo, F. de (2013). Sociología de la educación. Introducción al estudio de los fenómenos pedagógicos y de sus relaciones con los demás fenómenos sociales. México: Fondo de Cultura Económica.

Barato, S. (1998). Familia y comunidad. Bogotá: Universidad Santo Tomás.

Berger, P.y Luckmann, T. (1978). La construcción social de la realidad. Buenos Aires: Amorrortu.

Bezanilla, J. y Miranda, A. (2014). La familia como grupo social: una re-conceptualización. Alternativas en Psicología, 17(29), 58-73. Recuperado de http://pepsic.bvsalud.org/pdf/alpsi/v17n29/n29ao5.pdf

Bolívar, A. (2006). Familia y escuela: dos mundos llamados a trabajar en común. Revista de Educación, 339, 119146. Recuperado de https://www.researchgate.net/publication/28119472_Familia_y_escuela_dos_mundos_llamados_a_trabajar_en_comun

Bornand, M. (2011). Una indagación sobre los significados que los y las estudiantes construyen en torno a su Formación Politica en la Institución Escolar. Trabajo de Maestría, Universidad de Chile, Santiago.

Cebotarev, N. (1984). Nuevas perspectivas teóricas sobre el papel de la familia en el desarrollo. Manizález: Imprenta Cafetera.

Cuervo, A. (2010). Pautas de crianza y desarrollo socioafectivo en la infancia. Revista Diversitas, 6(1), 111-121. DOI: https://doi.org/10.15332/s1794-9998.2010.0001.08 
ISSN 0123-1294 | e-ISSN 2027-5358 | Educ.Educ. Vol. 22. No.3 | Septiembre-diciembre de 2019 | pp. 377-394.

Universidad de La Sabana | Facultad de Educación

Estrada, L. (2007). El ciclo vital de la familia. México: Ramdom House-Mondadori.

Frachia, C. (2015). Análisis de la dinámica familiar en adolescentes en conflicto con la ley desde un enfoque sistémico. Tesis de pregrado, Universidad de la República de Uruguay, Montevideo.

Froufe, S. (1995). El protagonismo de la familia en la transmisión de los valores sociales. Documentación Social. Revista de Estudios Sociales y de Sociología Aplicada, 98, 61-72.

Galeano, M. y Vélez, O. (2000). Investigación cualitativa: estado del arte. Medellín: Universidad de Antioquia.

Gallego, A., Pino, J. W., Álvarez, M., Vargas, E. y Correa, L. (2019). La dinámica familiar y estilos de crianza: pilares fundamentales en la dimensión socioafectiva. Hallazgos, 16(32), 131-149. DOI: https://doi. org/10.15332/2422409X.5093

Gallego, A. (2012). Recuperación crítica de los conceptos de familia, dinámica familiar y sus características. Revista Virtual Universidad Católica del Norte, 35, 326-345. Recuperado de http://www.redaly c.org/articulo. oa?id $=194224362017$

García, L.y López, R. (2014). La convivencia escoñar y la construcción de ciudadanía. Balance retrospectivo y desafíos del futuro. Bordón. Revista de Pedagogía,66(2), 93-106.DOI: http://doi.org/10.13042/Bordon.2014.66206

García, F., Martínez, K., Moreno, A.y Armenta, A. (2010). Un aparato teórico para el análisis de las representaciones de lo público en jóvenes universitarios. Civilizar, 10(19), 119-134. DOI: https://doi.org/10.22518/16578953.42

Gil, E. M. (2007). La familia: claves para una correcta gestión de las personas y situaciones familiares. Barcelona: Amat.

Infante, A. y Martínez, J. (2016). Concepciones sobre la crianza: el pensamiento de madres y padres de familia. Liberabit, 22(1), 31-41. DOI: https://doi.org/10.24265/liberabit.2016.v22n1.03

Jurado, M. (2013). Dinámica interna de las familias de los estudiantes que ejercen agresión física hacia sus compañeros. Un estudio desde el enfoque sistémico con familias de estudiantes de primaria de la institución educativa Alfonso Upegui Orozco. Trabajo de Maestría, Universidad Pontificia Bolivariana, Medellín, Colombia.

Louro, I. (2005). Modelo de salud del grupo familiar. Revista Cubana de Salud Pública, 31(4), 332-337. Recuperado el 27 de septiembre de 2018 , de http://scielo.sld.cu/scielo.php?script=sci_arttext\&pid=So864$34662005000400011 \& \operatorname{lng}=$ es \& tlng=es

Mendoza, L. et al. (2006). Análisis de la dinámica y funcionalidad familiar en atención primaria. Archivos en Medicina Familiar, 8(1), 27-32. Recuperado de https://www.medigraphic.com/cgi-bin/new/resumen. cgi?IDARTICULO=8430

Naval, C.y Ugarte, C. (2008). Educación para la ciudadanía y los Derechos Humanos, concepto clave en la ley de educación española. Revista Panamericana de Pedagogía, 11, 77-91. Recuperado de http://biblio.upmx.mx/ textos/roo10476.pdf 
Páez, G. (1984). Sociología de la familia. Bogotá: Universidad Santo Tomás.

Pino, J. W. (2012). Los conflictos y la dinámica interna familiar. Revista Momorias, 10(18), 159-170. Recuperado de https://revistas.ucc.edu.co/index.php/me/article/view/63/o

Restrepo, J. (2006). Estándares básicos en competencias ciudadanas: una aproximación al problema de la formación ciudadana en Colombia. Papel Político, 11(1) 137-175. Recuperado de http://www.scielo.org.co/scielo.php?script=sci_abstract\&pid=S0122-44092006000100006

Reusche, R. (2011). Dinámica psicológica de la familia. Temática Psicológica, 7(1), 7-16. Recuperado de http://www. unife.edu.pe/publicaciones/revistas/revista_tematica_psicologia_2011/reusche_lari.pdf

Rincón, M. (2012). La familia como escenario de socialización para la convivencia ciudadana. Eleuthera, 7, 116-132. Recuperado de http://eleuthera.ucaldas.edu.co/downloads/Eleuthera7_8.pdf

Robles, I. (2017). Dinámica familiar en madres solteras con hijas adolescentes. Trabajo de grado, Universidad Rafael Landivar, Guatemala.

Rojas, C. (2008). La construcción de ciudadanía en Colombia durante el gran siglo diecinueve 1810-1929. Paradigmas, 29, 295-333. Recuperado de https://www.researchgate.net/publication/277127596_La_construccion_de_la_ciudadania_en_Colombia_durante_el_gran_siglo_diecinueve_1810-1929

Rondón, L. (2011). Nuevas formas de familia y perspectivas para la mediación. El tránsito de la familia modelo a los distintos modelos familiares. I Congreso Internacional en Mediación y Conflictología: Cambios Sociales y Perspectivas de la Mediación para el Siglo XXI, coords. L. M. Rondón García y Eva Funes Jiménez (pp. 8193). Sevilla: Universidad Internacional de Andalucía.

Sánchez, G., Aguirre, M., Solano, N. y Viveros, E. (2015). Sobre la dinámica familiar. Revisión Documental, Cultura, Educación y Sociedad, 6(2), 117-138. Recuperado de https://revistascientificas.cuc.edu.co/culturaeducacionysociedad/article/view/1049

Sánchez, P. y Valdez, A. (2011). Una aproximación a la relación entre el rendimiento académico y la dinámica y estructura familiar en estudiantes de primaria. Revista Intercontinental de Psicología y Educación, 13(2), 177-196. Recuperado de https://www.redaly c.org/pdf/802/80220774009.pdf

Sautu, R., Boniolo, P., Dalle, P.y Elbert, R. (2005). Manual de metodología. Construcción del marco teórico, formulación de los objetivos y elección de la metodología. Buenos Aires: Clacso.

Serena, N. (1980). Antropología cultural. Adaptaciones socioculturales. México: Editorial Tipográfica.

Torres, A. et al. (2014). Dinámica familiary las prácticas de crianza como elemento clave en la educación parental. Tesis de grado, Universidad Nacional Abierta y a Distancia (UNAD), Bogotá. 
ISSN 0123-1294 | e-ISSN 2027-5358 | Educ.Educ. Vol. 22. No.3 | Septiembre-diciembre de 2019 | pp. 377-394.

Universidad de La Sabana | Facultad de Educación

Vásquez, C. (2005). Las nuevas tipologías familiares y los malestares interrelacionales que se suscitan en ellas. Revista Virtual. Universidad Católica del Norte, 14. Recuperado de http://revistavirtual.ucn.edu.co/index. php/RevistaUCN/article/view/251/

Vergara, L. (2017). Prácticas de crianza en la primera infancia en los municipios de Riosucio y Manzanares. Revista del Instituto de Estudios de Educación Universidad del Norte, 27, 22-33. DOI: http://dx.doi.org/10.14482/ zp.27.10980

Yabal, R. (2017). Características de la dinámica familiar de los pacientes con diagnóstico de depresión atendidos en consulta externa de un Hospital de Salud Mental. Lima, Perú 2016. Trabajo de Licenciatura en Enfermería, Universidad de Lima, Perú.

Zuluaga, J. (2004). LA familia como escenario para la construcción de ciudadanía: una perspectiva desde la socialización de la niñez. Revista Latinoamericana de Ciencias Sociales, Niñez y Juventud, 2(1), 127-148. Recuperado el 27 de septiembre de 2018, de http://www.scielo.org.co/scielo.php?script=sci_arttext\&pid=S1692$715 \times 2004000100005 \& \operatorname{lng}=e n \& \operatorname{tn} g=\mathrm{es}$ 